\title{
Frontières
}

\section{Perdre les siens, soigner les autres}

\section{Johanne de Montigny}

Volume 16, numéro 2, printemps 2004

Deuil, blessure vive

URI : https://id.erudit.org/iderudit/1074120ar

DOI : https://doi.org/10.7202/1074120ar

Aller au sommaire du numéro

Éditeur(s)

Université du Québec à Montréal

ISSN

1180-3479 (imprimé)

1916-0976 (numérique)

Découvrir la revue

\section{Citer cet article}

de Montigny, J. (2004). Perdre les siens, soigner les autres. Frontières, 16(2),

70-73. https://doi.org/10.7202/1074120ar

\section{Résumé de l'article}

Vivre une perte majeure peut amener une personne à s'investir profondément pour aider autrui à traverser des situations de perte. Les réflexions proposées à ce sujet s'alimentent à la parole échangée avec des intervenants en soins palliatifs. d'utilisation que vous pouvez consulter en ligne.

https://apropos.erudit.org/fr/usagers/politique-dutilisation/ 


\section{Résumé}

Vivre une perte majeure peut amener une personne à s'investir profondément pour aider autrui à traverser des situations de perte. Les réflexions proposées à ce sujet s'alimentent à la parole échangée avec des intervenants en soins palliatifs.

Mots clés: expérience de perte - soins palliatifs

\section{Abstract \\ When deeply affected by a loss, can we use this experience to better help the grieving persons? The author discusses this topic with caregivers in palliative care.}

Key words: loss experience - palliative care

\section{PERDRE LES SIENS, SOIGNER LES AUTRES}

\section{Johanne de Montigny, \\ psychologue, services de soins palliatifs, Centre universitaire de santé McGill, Hôpital Général de Montréal.}

Fortement inspirée d'un article intitulé « La sollicitude comme exorcisme » (Frappier, 1989), et à la suite de nombreuses années d'écoute de soignants qui participaient à des groupes hebdomadaires de parole, m'est venue l'idée de réfléchir et d'écrire sur la perte d'un proche comme élément clé parfois déclencheur de l'empathie, la compassion, l'engagement ou le souci de l'autre dans un contexte de soins prodigués aux malades en fin de vie, tantôt à titre d'intervenants professionnels dans des équipes pluridisciplinaires, de personnes significatives pour un grand malade ou de bénévoles mus par l'énergie du don relationnel.

Le don de soi comme exorcisme a fait l'objet d'une recherche en psychologie mettant en relation la sollicitude des soignantes avec la conscience qu'elles ont de la précarité de l'existence. "Ce ne sont pas tant les pertes en soi qui ont eu une importance déterminante dans leur choix ou leur intérêt pour œuvrer auprès des grands malades, mais plutôt l'aménagement qu'elles ont fait de leur vie en tenant compte à la fois des pertes et du besoin de les exorciser, c'est-à-dire de leur enlever leur potentiel dommageable, voire délétère » (Frappier, 1989, p. 27).

La nécessité d'agir en aidant les autres plutôt que de subir passivement et à long terme les difficiles répercussions du deuil provoquerait la quête d'une guérison personnelle puisée à même son élan naturel de partir à la rescousse des autres après avoir perdu les siens. Ainsi, pourrait-on surmonter le sentiment d'impuissance laissé par la perte en le troquant contre le sentiment d'utilité auprès d'étrangers à leur tour affligés par la maladie incurable.
Pour leur part, les bénévoles formeraient aussi un groupe de personnes désireuses de redonner à d'autres ce qu'elles auraient loupé lors de l'accompagnement d'un proche ou encore, et selon leurs dires, elles seraient mues par le désir de rendre grâce à l'autre en guise d'une reconnaissance infinie pour ce qu'elles ont reçu, c'est-à-dire une somme de gratifications personnelles obtenues lors d'une situation ultime. La découverte de moments forts et vécus dans l'intimité de la mort laisserait donc au survivant un apprentissage qu'il désire très souvent transmettre ou faire rayonner par le don de soi comme réaction ultérieure à la perte d'un proche.

Dans cette même perspective et en recueillant des propos des soignants (professionnels de la santé, intervenants psychosociaux, bénévoles et familles des grands malades), nous avons constaté, à titre de membres actifs en soins palliatifs, que la motivation liée au don de soi dans l'exercice d'un travail, d'une implication ou d'une responsabilité personnelle, le plus souvent émane effectivement d'une expérience de perte personnelle, significative et antérieure à l'émergence de l'énergie du don et du soin des autres. Plusieurs l'ont affirmé : cette connaissance de l'épreuve de la perte dicte l'envie de soigner.

Le Petit Robert définit la sollicitude comme une attention soutenue, à la fois soucieuse et affectueuse, son contraire étant l'indifférence. Or, en soins palliatifs, nous sommes des témoins privilégiés de la présence assidue de personnes serviables envers le malade, du relais indéfectible entre soignants toutes disciplines confondues et du sentiment de sécurité personnelle et collective qui s'en dégage. Par ailleurs, l'absence d'une présence rassurante dans les moments ultimes, c'est-à-dire la fuite d'un proche face à sa mort, ne serait pas forcément synonyme du désengagement 
personnel ou de son indifférence, mais elle pourrait refléter l'inconfort de l'humain face à son sentiment d'impuissance, à la peur de son incompétence affective et à l'incapacité de composer avec sa propre vulnérabilité, ou avec l'imprévu, c'est-à-dire avec son propre destin, incontournable, un destin personnel que nous réverbère la mort imminente de l'autre.

Personne n'est à l'abri de la souffrance liée à la perte, cependant, nous examinerons ici la décision de s'y exposer volontairement dans l'après-coup, telle une poussée naturelle, de contribuer concrètement à son apaisement, d'en tirer des leçons importantes et de créer du sens à partir de ces expériences fortes. La guérison personnelle proviendrait même de l'acte de soigner l'autre.

En nous accomplissant, nous donnons, en donnant, nous nous accomplissons. Plus nous sommes nous-mêmes, plus nous aidons les autres... En allant au fond de nous mêmes, nous touchons à la nature humaine qui se trouve en chacun et nous aidons donc les autres à prendre également contact avec leur force secrète, celle qui touche directement leur fragilité (Bertrand, 2000, p. 149).

Perdre un être cher ou significatif demeure une expérience parmi les plus éprouvantes. La perte même prévue ou attendue est étonnante par le vide qu'elle engendre en soi et autour de soi. À cause de sa force de frappe, la perte renvoie l'individu à sa solitude profonde. Une solitude qui paradoxalement l'isole de son besoin incommensurable et soudain de fraternité, d'affectivité et de chaleur humaine. Mais la solitude après la perte est aussi un état d'être et une expérience nécessaires. Elle est un préambule à la sollicitude. «La solitude constitue une aire de réflexion qui, bien utilisée, peut atténuer la douleur du départ » (Bureau, 1997, p. 23).

L'épreuve du deuil propulse le survivant dans une rencontre avec le néant, l'absence, le vide, la rupture, la solitude. Dans les premiers temps suivant l'expérience de la perte, le caractère passager de nos investissements affectifs ou l'incapacité de les récupérer par le biais d'un mémorial immédiatement accessible se vit comme une prise de conscience catastrophique, un traumatisme insurmontable. De plus, le dur sentiment d'avoir raté l'occasion d'aimer mieux ou d'être enfin aimé ajoute au vide personnel et existentiel. Voilà ce que nous révèle aussi la clinique du deuil.

Pour contrer la douleur de la perte, et après un temps de repli sur soi, l'homme cherche alors différentes astuces l'entrâ̂nant dans un espace de guérison person- nelle. L'esprit du don (Godbout, 1992, p. 31) se présente comme une voie d'échappement à la prolongation de sa propre douleur. Ainsi, cet élan du cœur ou de survie personnelle se traduira-t-il par le souci de l'autre, l'altruisme ou le don relationnel prodigué à un étranger, histoire de larguer la perte du connu.

Dans pareilles circonstances, il s'agirait $\mathrm{du}$ don authentique qui, selon Godbout, suppose un altruisme véritable. Sa réflexion à propos de la gratuité ou non du don, ou du don comme remboursement d'une dette personnelle, incite le lecteur à approfondir ses motivations dans l'art de donner aux autres ce qui lui a été retiré. L'hypothèse de l'auteur repose sur le fait que le désir de donner soit aussi important que celui de recevoir. "Que donner, transmettre, rendre, que la compassion et la générosité sont aussi essentiels que prendre, s'approprier ou conserver, que l'envie ou l'égoïsme. »

Bien que Godbout et son collaborateur nous amènent à comprendre que le don n'est pas toujours gratuit, c'est-à-dire sans attente de réciprocité, nous nous concentrons ici sur le désir spécifique du soignant de retourner une parcelle de ce qu'il a reçu (la dette du survivant - pourrait-on le dire ainsi) dans ses expériences de pertes et de gains antérieurs.

Il s'agirait en quelque sorte de l'héritage affectif, un legs que nous laissent ceux qui meurent avant nous, une notion très forte sur l'importance relationnelle, communicationnelle et spirituelle que le survivant pourra surtout découvrir et apprécier au moment de perdre un être cher. Ultérieurement,

la motivation de loin la plus importante qui ressort pour expliquer l'engagement, par exemple, dans l'action volontaire, c'est le fait qu'on a beaucoup reçu : de sa famille, de son milieu, de la vie en général... Quoi qu'il en soit, les personnes qui font de l'action volontaire le font par plaisir et retirent plus que ce qu'elles donnent, même, chez les bénévoles, c'est-à-dire dans les groupes où le service rendu est unilatéral et non réciproque...

Aussi le bénévole dira-t-il : ce n'est pas par grandeur d'âme que j'agis, je reçois tellement des gens que j'aide

(Godbout, 1992, p. 108-109).

C'est sans nul doute dans ce sens que l'on persiste à croire que le don n'est pas gratuit, car tous ceux qui donnent ne se lassent pas de dire combien ils reçoivent en donnant. Le sentiment de redevance, une dette nécessaire à rembourser, serait l'un des motifs évoqués par un grand nombre de personnes préoccupées à bien servir les autres.
Dans l'Amour ultime (de Hennezel et de Montigny, 1990, p. 152-153), Marie de Hennezel nous rapporte les résultats de sa recherche sur les motivations des soignants en soins palliatifs, les principales étant «Vivre des temps forts; et relever un défi. Il s'agit d'opérer une sorte de saut, de déplacement vers le haut... de transformer une catastrophe en événement plein de sens, d'accéder au symbolique ». Car à quoi donc servirait la souffrance sans notre capacité de la transcender, la transformer et l'intégrer dans le cadre d'un apprentissage exceptionnel au cœur de sa propre vie?

Or le don de soi à des personnes étrangères serait aussi, fort possiblement, symbolique du manque à gagner de la personne proche d'ores et déjà décédée, c'est-à-dire la personne qui nous a quittés trop tôt ou trop tard sans que l'on ait pu réagir promptement, avant même d'avoir pu lui donner le meilleur de soi tout juste avant son départ. Dans ce sens, la symbolique à retracer dans le don du soignant ou dans l'implication du bénévole aurait trait à la représentation que l'on se fait de l'être cher disparu en redonnant de sa personne mais cette fois à un étranger, à son remplaçant, en l'occurrence le nouveau mourant. L'expérience personnelle de la perte rendrait ainsi le survivant hyper-conscient d'une vie qui tire à sa fin. Et c'est pourquoi il se promet en réaction à sa dure épreuve de ne plus jamais rater le rendez-vous terminal désormais remis en scène par l'étranger.

Deux thèmes sous-tendent le discours des soignants dans leur penchant pour la sollicitude : le désir de réparer les manques expérimentés dans leur vie personnelle et le sentiment héroïque de survivre à la mort des autres au quotidien. La dure impression d'avoir en cours de route failli à la tâche exigeante de soigner un proche, par exemple un parent, entraînerait un sentiment de culpabilité difficile à déloger autrement que par la réparation de cet échec en aidant les autres. Symboliquement, cette attitude reflète une demande de pardon par le travail de la sollicitude.

Plutôt que de subir une mort psychique en conséquence de la mort physique d'un être cher ou de se morfondre dans un deuil non résolu pour des raisons de fuite personnelle à un moment aussi crucial, le survivant décidera de lui-même, parfois consciemment parfois inconsciemment, de se confronter à nouveau et ultérieurement à la mort via l'accompagnement de l'étranger qui s'apprête à mourir. Ainsi, dans plusieurs cas, participer de façon répétée à la scène du mourir permettrait-il au soignant de réparer son passé tout en conservant l'impression d'y survivre héroïquement au quotidien. Il offrirait alors son ancrage à celui qui meurt en éprouvant en quelque 
sorte le sentiment héroïque d'avoir échappé au siphon de la mort sans pour autant et à l'avenir s'enfuir devant elle. La réparation est une façon créatrice de contrer la menace à son intégrité.

Dans son mémoire de maîtrise, Denis René (1988, p. 34) se réfère à la théorie de Mélanie Klein pour décrire la « réparation réussie »dont la principale source se retrouve incidemment dans les manifestations de la sollicitude, ainsi qu'à celle de Winnicott pour expliquer que la sollicitude serait l'expression même de la réussite de l'organisation du moi. L'auteur de cette recherche sur la sollicitude formule toutefois une mise en garde :

La motivation à aider les autres, à se soucier de leur bien-être physique et psychologique, est dite authentique lorsqu'elle est issue d'un désir plus ou moins inconscient de la personne de s'assurer une vie intérieure stable, de partager son propre bien-être et de manifester la responsabilité qu'elle ressent envers les autres.

Autrement dit, si le don de soi est calculé en fonction de la seule attente des autres pour assurer son équilibre personnel, la démarche sera une fois de plus vouée à un échec, du moins au sentiment d'avoir échoué. Les bénéfices secondaires de la sollicitude ne doivent ni être préconçus, planifiés ou attendus. Quoi donc alors espérer? Le soignant pourra ressentir sa joie profonde d'aider en l'exprimant par l'intermédiaire de la pensée de Bobin (1997, p. 79, 96) : «C'est l'imprévu que j'espère, et lui seul. Partout, toujours. Dans les plis d'une conversation, dans le gué d'un livre, dans les subtilités d'un ciel. Je le guette autant que je l'espère. Ce à quoi je ne m'attends pas, c'est cela que j'attends. Et ce que je trouve est mille fois plus beau que ce que je cherche. » Voilà dans quel esprit le soignant pourrait aborder le soigné.

La sollicitude donne un sens à la perte et le sens reste toujours à découvrir. C'est dire que l'effet tonique de l'accompagnement ne sera pas le même pour chacun. Parce que la signification de son implication personnelle porte sa symbolique singulière, elle est toujours en lien avec son histoire d'amour, de perte et de restauration personnelle. Mais le travail de guérison par la sollicitude se joue d'abord à son insu car le legs des mourants est un cadeau que l'on ne cesse de développer tout au fond de soi, au fil de sa propre survie.

La perte agit comme le détonateur de la compassion, l'éveil de sa sensibilité à l'égard de la peine d'autrui.

La compassion, c'est-à-dire étymologiquement souffrir avec l'autre, désigne une qualité de la relation par laquelle on lui manifeste que l'on ressent ce qu'il vit... Dans la compassion, se manifeste une forme de présence et de communion qui est en définitive la seule façon d'aimer autrui, quand il est dans le malheur...; il n'y a pas de compassion sans affection... La fonction réparatrice de la compassion réside dans la consolation (Fischer, 2003, p. 189-190).

C'est donc la souffrance liée à la perte d'un proche qui avant tout ouvre une brèche sur sa capacité de vivre seul, s'entend dans une solitude profonde, avec un sentiment d'abandon à soi avant de reprendre le chemin de sa vie bouleversée. Son écart des autres, le repli sur soi, ce temps voué à l'isolement, sont des phases nécessaires et incontournables avant de retrouver son espace vital auprès d'autrui. Impossible, voire dangereux, de sauter à pieds joints dans l'appât de la sollicitude sans avoir franchi les ténèbres de son propre deuil. Le risque est trop grand de s'y perdre ou de confondre l'altruisme avec l'étanchement de ses propres besoins. Ce sont là des écueils importants que certains soignants ou bénévoles n'ont pas su identifier avant de se consacrer au service des autres.

«La bonté est suspecte! » telle fut la réflexion d'une soignante pour qui la sollicitude renferme implicitement le besoin de combler un vide personnel. Certaines personnes n'ont pas à ce jour expérimenté la force d'un amour inconditionnel, désintéressé, c'est-à-dire sans attente de réciprocité. Faut-il d'abord l'avoir reçu dans un moment crucial et inattendu avant de le prodiguer à son tour? Possiblement. Nos croyances ne sont-elles pas le produit de nos expériences?

De quelle façon aidons-nous les autres face à leur maladie incurable ? Quel est notre rapport au silence, au chagrin, à la colère manifeste ou passive de l'autre ? Sommes-nous capables d'offrir une écoute pure, une présence rassurante, une posture d'humilité face à l'intimité que la mort imminente suscite dans la rencontre? L'intimité de la rencontre et sa singularité. Voilà ce qui décrit l'ambiance de la chambre d'un grand malade. Comment y entrer, y rester, s'y retirer ? Autrement dit, comment composer avec les paradoxes de l'attachement sain et du détachement nécessaire, impératif ? Comment à la fois saluer l'autre avec un accueil sur mesure et lui manifester l'importance d'un au revoir accompli ? Enfin, comment porter les questions d'autrui sans pour autant tomber dans le piège d'y répondre assurément?

Elle est difficile à décrire cette forme de spiritualité... car il s'agit bien là d'une rencontre spirituelle, et elle porte en elle des sous-thèmes et des références propres à chacun. Pour certains, la spiritualité s'applique à donner un sens à l'existence, pour d'autres, elle vise la qualité relationnelle, l'amour inconditionnel, l'espérance, la communion ou la fusion avec la création du monde. " $\mathrm{Ne}$ sais-tu pas que le ciel est en toi ? ", nous dirait Singer (s.d., p. 13) : " Il est essentiel de prendre soin de ce ciel en nous, invisible aux autres, de ce sanctuaire que la vie nous a édifié et que peuplent tous les intercesseurs, les messagers, ceux qui, de façons multiples, conduisent vers le meilleur de nous-mêmes. »

Car celui qui meurt fait appel au meilleur de nousmêmes, et c'est bien là que se situe la plus grande découverte sur soi. Ce désir de déployer ses talents, de créer du beau, de partager son avoir, de se soucier de l'être devant soi, se multiplie devant un frère démuni. Or, un tel don de soi provient d'une source d'énergie inaccessible dans «la vie ordinaire », car auprès du mourant on ne peut parler de vie ordinaire, elle tire à sa fin. Tenir la main du mourant rend bien compte de la précarité du geste. Le moment devient sacré à cause de sa fragilité, sa fugacité. La proximité avec une personne mourante secoue le survivant, l'entraîne dans une réflexion sur le court passage de ceux et celles qui l'entourent, l'influence, le pousse à vivre encore mieux la suite. C'est tout cela qui n'est pas gratuit, puisque le prix à payer, offrir sa présence à la future absence, représente tout un travail sur soi avant d'entreprendre l'aide destinée aux autres.

Revenons à l'article intitulé «La sollicitude comme exorcisme» (Frappier, 1989). L'auteur, Paul Frappier, nous interpelle : « Le choix de vivre ces situations limites - où on se donne tout en sachant que cela nous expose au déchirement - ne serait-il pas une manière de vouloir vivre plus intensément ou à tout le moins plus authentiquement ? »

La question n'est pas banale. S'il y a un prix à payer, le détachement et la perte, dans l'accompagnement de celui qui meurt, il y a également un prix à payer dans l'évitement de moments forts : vivre à la surface des choses, rater les profondeurs du potentiel humain. 


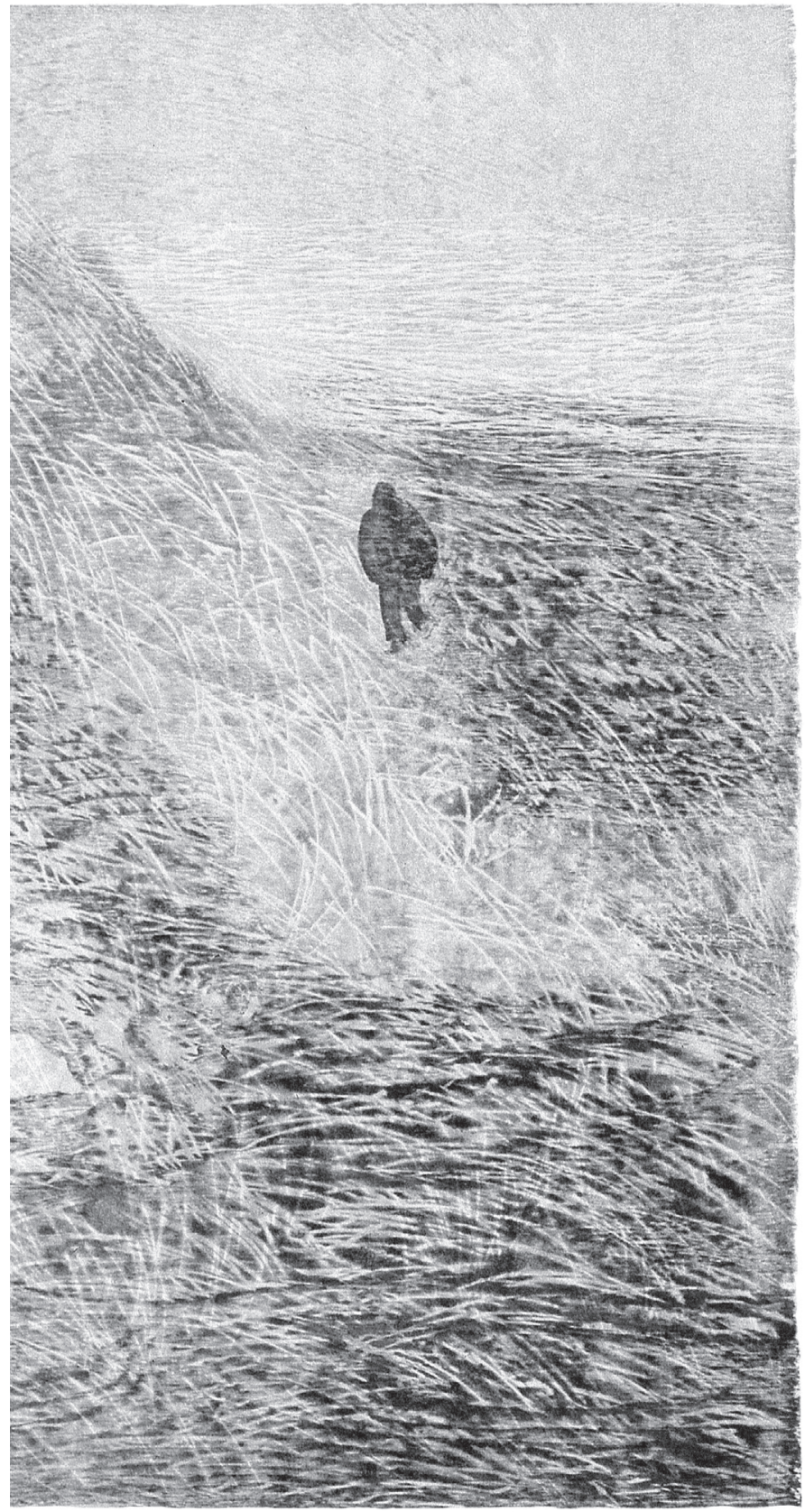

possible dans les milieux où les soignants doivent composer avec les limites du temps alloué au service de chacun, le manque de personnel et les restrictions budgétaires ? Dans le contexte actuel, et pour la plupart des équipes de travail, l'accomplissement des tâches reliées aux soins triomphe de l'attention portée aux malades. La qualité relationnelle est considérée comme un privilège, voire un luxe, difficilement accessible en dehors des unités de phase terminale. Le témoignage des patients et de leurs proches en font foi.

Ainsi, le soignant qui opte pour la fluidité relationnelle, l'intelligence émotionnelle et la découverte d'un sens à la souffrance, penchera vers le milieu des soins palliatifs. Cette qualité de contact est une valeur que tout intervenant en soins palliatifs s'efforce de préserver et de défendre dans un monde où les contacts virtuels risquent de se substituer à l'intimité. À la fin de la vie, l'être humain se voit dépossédé de sa santé globale, de son rôle social, de ses rêves, de son énergie vitale. Seul le lien avec une personne significative peut demeurer, voire s'agrandir jusqu'à la fin et, symboliquement, survivre à la mort. C'est pourquoi la qualité relationnelle prédomine dans

Cette qualité relationnelle est peu accessible en dehors de l'expérience de l'investissement personnel et de la perte qui s'ensuit. En fait, la perte nous permet d'évaluer ce qui fut, ce que l'on est et ce qui reste à découvrir.

Comment serait-il autrement possible d'éprouver la gratitude et la générosité ?

Tous les intervenants qui œuvrent auprès des personnes en phase terminale n'affirment peut-être pas un lien direct ou strict entre l'impact d'une perte personnelle et leur implication auprès des mourants et des familles qui les accompagnent. Cependant, tous les participants aux groupes de parole décrivent leur milieu de travail comme un terrain exceptionnel pour établir des contacts authentiques, réfléchir sur sa propre mort et bien comprendre son propre rapport à la vie.

Ce désir d'être vrai, d'être soi et d'entrer en relation profonde avec l'autre est-il toires : la mort arrive bel et bien aux autres, il en est un témoin au quotidien ; cependant, ne risque-t-elle pas de le happer à son tour ? Hyper-conscient de sa proximité ?

La question fondamentale du soignant s'exprime alors ainsi : mon travail de sollicitude me permet-il d'apprivoiser ma propre mort, de mieux m'y préparer ? Et la réponse d'un pair s'ensuit : difficile de l'affirmer, cependant, la riche expérience nous permettra de bonifier notre façon de vivre.

Côtoyer la mort au quotidien fait appel à une certaine sagesse, celle de s'installer au cœur d'une relation profonde mais brève et de cumuler le souvenir des temps forts en compensation de la permanence des absences. Il faut beaucoup aimer la vie pour tolérer la vue de la mort. Il faut aussi comprendre que la perte est une étape éprouvante mais que sans elle, il nous serait bien plus difficile de glorifier les acquis.

Le travail en soins palliatifs offre un espace de réflexion sur le sens de sa présence au monde qui le plus souvent se fonde sur son désir ardent de bien servir un plus souffrant que soi. Vivre à fond la rencontre terminale promet une expérience à la fois troublante et gratifiante.

\section{Bibliographie}

BERTRAND, Pierre (2000). Éloge de la fragilité, Montréal, Liber.

BOBIN, Christian (1997). Autoportrait au radiateur, Paris, Gallimard.

BUREAU, Jules (1997). Le goût de la solitude, Montréal, collection «Psycho-Santé».

DE HENNEZEL, Marie et Johanne DE MONTIGNY (1990). L'Amour ultime (psychologie et tendresse dans la traversée du mourir), Montréal, Stanké.

FISCHER, Gustave-Nicolas (2003). Les blessures psychiques (La force de revivre), Paris, Odile Jacob.

FRAPPIER, Paul (1989). «La sollicitude comme exorcisme », Frontières, vol. 1, n 3 (hiver), p. 26-29.

GODBOUT, Jacques T. (1992). L'esprit du don, en collaboration avec Alain Caillé, Paris, La Découverte.

RENÉ, Denis (1988). Étude phénoménologique exploratoire des manifestations de sollicitude en regard des structures de l'imaginaire chez des intervenants auprès de malades en situations létales, Mémoire de maîtrise en psychologie, Université du Québec à Montréal.

SINGER, Christiane (s.d.). Où cours-tu ? Ne sais-tu pas que le ciel est en toi ?, Paris, Albin Michel. 